\title{
Preventive Replacement Policy and Imperfect Maintenance Model with Random Working Times
}

\author{
Chin-Chih Chang \\ Department of Chains and Franchising Management, \\ Takming University of Science and Technology, \\ Taipei, Taiwan \\ ccchang@takming.edu.tw
}

\begin{abstract}
This article proposes the preventive replacement policy for an operating system which may continuously work for $\mathbf{N}$ jobs with random working times and be imperfectly maintained upon failure. As a failure occurs, the system suffers one of the two types of failures based on some random mechanism: type-I (repairable or minor) failure is rectified by a minimal repair, or type-II (non-repairable or catastrophic) failure is removed by a corrective replacement. A bivariate replacement policy is considered in which the system is replaced preventively at an operating time $T$, at number $N$ of working times, or replaced correctively after any type-II failure, whichever occurs first. The optimal schedule of preventive replacement that minimizes the mean cost rate is presented theoretically and determined numerically. Because the framework and analysis are general, the proposed model extends several existing results.
\end{abstract}

Keywords-preventive replacement; imperfect maintenance; random working time; optimization; reliability

\section{INTRODUCTION}

Various kinds of systems are subjected to aging and degradation after being launched and suffer stochastic failures during actual operation. For the non-repairable system, replacement is carried out once it fails; while most repairable systems, such as aircrafts, power generators, nuclear systems, and computer systems, can be restored to a functioning state through a specific maintenance activity. Therefore, selecting cost efficient, effective maintenance actions and strategies to improve system reliability and physical performance is crucial work in reliability engineering.

To measure the quality of maintenance activities, one can classify maintenance into some categories according to its impact on the system. In most cases, maintenance does not make a system "as good as new" (perfect maintenance or simply replacement) or "as bad as old" (minimal maintenance or minimal repair). A minimal repair restores the system to its functioning condition just prior to the failure, and the failure rate is not affected by minimal repair [1]. However, it is more realistic to consider that maintenance restores a system to somewhere between these two possible extreme cases, and that activity is referred to as imperfect maintenance. Various models have been proposed for imperfect maintenance in the past from different perspectives as reviewed in complete detail in [2]. In particular, one probabilistic approach of modeling an imperfect maintenance activity is the choice between replacement and minimal repair based on some random mechanism. Brown and Proschan [3] considered a model in which, upon failure, the system is replaced with probability $p$ and is minimally repaired with probability $q(=1-p)$, independently of the previous history of maintenance. Further enhancement to this probabilistic approach of two failure mechanisms was to consider the probabilities as timedependent functions [4]. These results have been extended and applied extensively in reliability research, some recent applications in such as cumulative repair-cost limit replacement policy and used system can be found in [5] - [7], respectively. In this paper, we are concerned with constructing the preventive replacement policy of using the imperfect maintenance of two failure mechanisms.

Preventive replacement actions for an operating system are generally grouped into time-based replacement that is based on planned time, age, or usage time of the system, etc. Age replacement policy (ARP) is a well-known preventive replacement model of unique time scale: an operating system is replaced at age $T$ or at failure, whichever occurs first [1]. However, for some systems in offices and industry, when a job has a variable working cycle, it would be better to do maintenance or replacement after it has completed its work [8]. If a system is replaced only at random working times, the policy can be called a random replacement policy. The early investigation into the random replacement policy can be found in [9] - [10]. Recently, replacement policies with two time scales have been discussed and proposed [11]. Chen et al. [12] take up an operating system which works at successive random times and its age replacement policies. Chen et al. [13] consider replacement and maintenance policies for an operating system which works at random times and undergoes minimal repair at failures. In this paper, we will further develop the modified random and age replacement policies for an operating system with jobs successively and imperfect maintenance quality.

\section{PREVENTIVE REPLACEMENT}

In this article, a modified preventive replacement policy with working times for a system in which repair, maintenance, and replacement take place according to the following scheme. 
- A new system with a failure time $X$ begins to operate at time 0 . When $X$ has a general distribution $F(t)$ and probability density function $f(t)$, then the failure rate $r(t) \equiv f(t) / \bar{F}(t)$ is assumed to increase to. A preventive replacement (PR) is planned to carry out when the system reaches age.

- It is assumed that the system failures at time $t$ can experience two types: a type-I failure (repairable or minor) occurs with probability $q$ and is corrected by a minimal repair, whereas a type-II failure (nonrepairable or catastrophic) occurs with probability $p(=1-q)$ and requires a corrective replacement (CR).

- It is assumed that $Y_{j}$ is the $j$ th working time of the system and is independent with an identical distribution $G(t) \equiv P\left(Y_{j} \leq t\right)$. Then the total working time $S_{j} \equiv Y_{1}+\cdots+Y_{j}$ up to the $j$ th working time has a distribution $G^{(j)}(t) \equiv P\left(S_{j} \leq t\right)$, where $G^{(j)}(t)$ is the $j$-fold Stieltjes convolution of $G(t)$ with itself and $G^{(0)}(t) \equiv 1$. Another $\mathrm{PR}$ is performed at the completion of the $N$ th working time.

- In summary, PR is performed before any type-II failures at age $T$ or at number $N$ of working times, whichever occurs first, which is called preventive replacement first (PRF). CR is done immediately after any type-II failure.

- Repairs and replacements are completed instantaneously. After a replacement, the system becomes brand new and resets to time 0 . A renewal cycle is defined as the time interval between two consecutive replacements.

Let $Z$ be the waiting time until the first type-II failure. From Beichelt (1993), the survival function of $Z$ is directly obtained

$$
\bar{F}_{p}(t) \equiv P(Z>t)=\exp (-p \Lambda(t))
$$

where the cumulative hazard $\Lambda(t) \equiv \int_{0}^{t} r(u) \mathrm{d} u=-\ln \bar{F}(t)$ is the mean number of failures that occur in $[0, t]$. Te mean time of a renewal cycle can be given

$$
\int_{0}^{T} \bar{F}_{p}(t)\left[1-G^{(N)}(t)\right] \mathrm{d} t
$$

Next, because the mean number of type-I failures (minimal repairs) in $[0, t]$ can be derived as $\Lambda_{q}(t) \equiv q \Lambda(t)$ [14], the total mean number of type-I failures before replacement is

$$
\int_{0}^{T} \bar{F}_{p}(t)\left[1-G^{(N)}(t)\right] q r(t) \mathrm{d} t
$$

Furthermore, the following cost structure is introduced for this model. The PR costs due to age $T$ and due to number $N$ are $C_{T}$ and $C_{N}$, respectively. The CR cost due to type-II failure is $C_{Z}$. It is also assumed that the corrective replacement cost is not less than the preventive replacement costs. Cost $c_{m}$ is the minimal repair cost. Then, the mean cost rate of a renewal cycle is, from (2)-(3),

$$
\begin{gathered}
C_{T}+\left(C_{N}-C_{T}\right) \int_{0}^{T} \bar{F}_{p}(t) \mathrm{d} G^{(N)}(t) \\
J(T, N) \equiv \frac{+\left(C_{Z}-C_{T}\right) \int_{0}^{T}\left[1-G^{(N)}(t)\right] \mathrm{d} F_{p}(t)+c_{m} \int_{0}^{T} \bar{F}_{p}(t)\left[1-G^{(N)}(t)\right] q r(t) \mathrm{d} t}{\int_{0}^{T} \bar{F}_{p}(t)\left[1-G^{(N)}(t)\right] \mathrm{d} t} .
\end{gathered}
$$

\section{OPTIMIZATION}

First, we derive an optimal number $N^{*}$ which minimizes $J(T, N)$ with respect to $N$ for a given $T$. We see that the inequalities $J(T, N+1) \geq J(T, N)$ and $J(T, N)<J(T, N-1)$ hold if and only if

$$
L(N ; T) \geq C_{T} \text { and } L(N-1 ; T)<C_{T},
$$

where

$$
\begin{aligned}
& L(N ; T) \equiv\left\{\begin{array}{l}
\zeta(N ; T) \int_{0}^{T} \bar{F}_{p}(t)\left[1-G^{(N)}(t)\right] \mathrm{d} t-\left\{\left(C_{N}-C_{T}\right) \int_{0}^{T} \bar{F}_{p}(t) \mathrm{d} G^{(N)}(t)\right. \\
\left.+\left(C_{Z}-C_{T}\right) \int_{0}^{T}\left[1-G^{(N)}(t)\right] \mathrm{d} F_{p}(t)+c_{m} \int_{0}^{T} \bar{F}_{p}(t)\left[1-G^{(N)}(t)\right] q r(t) \mathrm{d} t\right\}, \\
\text { for } n=1,2, \cdots, \\
0, \text { for } n=0,
\end{array}\right. \\
& \quad\left(C_{T}-C_{N}\right) \int_{0}^{T} \bar{F}_{p}(t) \mathrm{d} \tilde{G}^{(N)}(t) \\
& \zeta(N ; T) \equiv \frac{+\left(C_{Z}-C_{T}\right) \int_{0}^{T} \tilde{G}^{(N)}(t) \mathrm{d} F_{p}(t)+c_{m} \int_{0}^{T} \bar{F}_{p}(t) \tilde{G}^{(N)}(t) q r(t) \mathrm{d} t}{\int_{0}^{T} \bar{F}_{p}(t) \tilde{G}^{(N)}(t) \mathrm{d} t},
\end{aligned}
$$

and $\tilde{G}^{(N)}(t) \equiv G^{(N)}(t)-G^{(N+1)}(t)$.

Furthermore, let

$$
\rho \equiv \frac{C_{T}+\left(C_{z}-C_{T}\right) F_{p}(T)+c_{m} \int_{0}^{T} \bar{F}_{p}(t) q r(t) \mathrm{d} t}{\int_{0}^{T} \bar{F}_{p}(t) \mathrm{d} t} .
$$

The optimal policy $N^{*}$ that minimizes the mean cost rate $J(T, N)$ with respect to $N$ for a given $T$ can be summarized as below. 
If $\zeta(N ; T)$ is increasing in $N$ and $\lim _{N \rightarrow \infty} \zeta(N ; T)>\rho$, then there exists a finite and unique $N^{*}$ that satisfies $L\left(N^{*} ; T\right) \geq C_{T}$ and $L\left(N^{*}-1\right)<C_{T}$ for $N^{*}=1,2, \cdots$.

Next, we derive an optimal age $T^{*}$ that minimizes $J(T, N)$ with respect to $T$ for a given $N$. Differentiating $J(T, N)$ in (4) with respect to $T$ and setting it equal to zero, we see that $\partial J(T, N) / \partial T=0$ if and only if

$$
Q(T ; N)=C_{T},
$$

where

$$
\begin{aligned}
& \begin{aligned}
Q(T ; N) \equiv & \varphi(T ; N) \int_{0}^{T} \bar{F}_{p}(t)\left[1-G^{(N)}(t)\right] \mathrm{d} t-\left[\left(C_{N}-C_{T}\right) \int_{0}^{T} \bar{F}_{p}(t) \mathrm{d} G^{(N)}(t)\right. \\
& \left.+\left(C_{Z}-C_{T}\right) \int_{0}^{T}\left[1-G^{(N)}(t)\right] \mathrm{d} F_{p}(t)+c_{m} \int_{0}^{T} \bar{F}_{p}(t)\left[1-G^{(N)}(t)\right] q r(t) \mathrm{d} t\right]
\end{aligned} \\
& \varphi(T ; N) \equiv\left(C_{N}-C_{T}\right) r_{G_{N}}(T)+\left(C_{Z}-C_{T}\right) r_{p}(T)+c_{m} q r(T), \\
& \text { and } r_{\sigma_{N}}(T) \equiv \frac{\partial G^{(N)}(T) / \partial T}{1-G^{(N)}(T)}, r_{p}(T) \equiv \frac{\partial F_{p}(T) / \partial T}{\bar{F}_{p}(T)}
\end{aligned}
$$

Furthermore, let

$$
\begin{gathered}
C_{T}+\left(C_{N}-C_{T}\right) \int_{0}^{\infty} \bar{F}_{p}(t) \mathrm{d} G^{(N)}(t) \\
\theta \equiv \frac{+\left(C_{z}-C_{T}\right) \int_{0}^{\infty}\left[1-G^{(N)}(t) \mathrm{d} F_{p}(t)+c_{m} \int_{0}^{\infty} \bar{F}_{p}(t)\left[1-G^{(N)}(t)\right] q r(t) \mathrm{d} t\right.}{\int_{0}^{\infty} \bar{F}_{p}(t)\left[1-G^{(N)}(t)\right] \mathrm{d} t} .
\end{gathered}
$$

We can summarize the structure properties of the optimal policy $T^{*}$ that minimizes $J(T, N)$ with respect to $T$ for a given $N$ as follows.

Suppose that $\varphi(T ; N)$ is continue and increasing in $T$. If $\lim \varphi(T ; N)>\theta$, then there exists a finite and unique $T^{*}$ which satisfies (9) and the optimal mean cost rate is $J\left(T^{*}, N\right)=\varphi\left(T^{*} ; N\right) \quad ;$ otherwise, the optimal policy is $T^{*} \rightarrow \infty$.

\section{NUMERICAL EXAMPLE}

In this section, a numerical example is given to illustrate the characteristics of the presented models. Suppose that a system with a failure time $X$ deteriorates increasingly with its age, and fails according to the Weibull distribution $F(t) \equiv P(X \leq t)$ with scale parameter $\alpha$ and shape parameter $\beta$, i.e., $F(t)=1-\exp \left[(-\alpha t)^{\beta}\right]$. We assume that each random working cycle of the system has an independent exponential distribution $G(t)=1-\exp (-\theta t)$.

In practice, the repair cost is a random variable and the decision to repair or replace a failed system may depend on the estimated repair cost, denoted by $C$. Such a formulation has been studied by [15]-[16], and is central to what is called repair limit replacement policy. Here, we consider a repair limit replacement policy where, in a failure state, one replaces the system or repairs it depending on the random cost $C$. A system undergoes a corrective replacement if $C>\delta c_{\infty}$ and is minimally repaired if $C \leq \delta c_{\infty}$, where $c_{\infty}$ is the constant cost of replacement at failure and $\delta(0 \leq \delta \leq 1)$ can be interpreted as a fraction of the constant $\operatorname{cost} c_{\infty}$. Suppose that the random repair cost $C$ has a distribution $L(u)$ and density function $l(u)$. Hence, $\delta$ satisfies $q=\int_{0}^{\delta c_{c_{\infty}}} l(u) \mathrm{d} u$, and the expected minimal repair cost $c_{m}$ can be given by $c_{m}=\int_{0}^{\delta c_{m}} u l(u) \mathrm{d} u / q$. The parameter $q$ (minimal repair probability) is varied to determine its influence on the optimal policies.

The mean cost rate $J(T, N)$ in (4) is a bivariate function of $(T, N)$, and we may obtain the bivariate optimal replacement

\begin{tabular}{|c|c|c|c|c|c|c|c|c|c|}
\hline \multirow[b]{2}{*}{$q$} & \multicolumn{3}{|c|}{$\begin{array}{c}\text { Case } 1 . \\
C_{T}=100, C_{Y}=150\end{array}$} & \multicolumn{3}{|c|}{$\begin{array}{c}\text { Case 2. } \\
C_{T}=150, C_{Y}=200\end{array}$} & \multicolumn{3}{|c|}{$\begin{array}{c}\text { Case } 3 . \\
C_{T}=200, C_{Y}=250\end{array}$} \\
\hline & $N$ & $T^{*}$ & $J(T, N)^{*}$ & $N$ & $T$ & $J(T, M)^{*}$ & $N$ & $T^{*}$ & $J(T, M)^{*}$ \\
\hline 0.9 & 25 & 7.5820 & 26.63 & 29 & 9.4456 & 32.23 & 32 & 11.1009 & 36.7700 \\
\hline 0.8 & 23 & 6.3286 & 32.0233 & 27 & 7.9384 & 38.5814 & 29 & 9.3993 & 43.8019 \\
\hline 0.7 & 21 & 5.5357 & 36.6809 & 25 & 6.9690 & 44.0878 & 27 & 8.2848 & 49.9263 \\
\hline 0.6 & 21 & 4.9770 & 40.8560 & 24 & 6.2795 & 49.0236 & 26 & 7.4836 & 55.4305 \\
\hline 0.5 & 19 & 4.5562 & 44.6532 & 23 & 5.7569 & 53.5426 & 25 & 6.8721 & 60.4786 \\
\hline 0.4 & 19 & 4.2243 & 48.1865 & 22 & 5.3430 & 57.7415 & 24 & 6.3854 & 65.1755 \\
\hline 0.3 & 19 & 3.9538 & 51.5032 & 21 & 5.0045 & 61.6869 & 23 & 5.9859 & 69.5933 \\
\hline 0.2 & 18 & 3.7275 & 54.6455 & 21 & 4.7206 & 65.4280 & 22 & 5.6497 & 73.7864 \\
\hline 0.1 & 17 & 3.5340 & 57.6485 & 20 & 4.4773 & 69.0063 & 22 & 5.3610 & 77.8008 \\
\hline 0.0 & 16 & 3.3645 & 60.5612 & 20 & 4.2636 & 72.4815 & 21 & 5.1065 & 81.7048 \\
\hline
\end{tabular}
policy $(T, N)^{*}$ such that $J(T, N)^{*}=\min _{N}\left[\min _{T} J(T, N)\right]$. The optimal preventive replacement policies and related minimum mean cost rates for the imperfect maintenance models under different minimal repair probabilities are reported in Table I.

TABLE I. OPTIMAL PREVENTIVE REPLACEMENT POLICIES AND MEAN COST RATES

From the numerical results, we have the following observations:

- We see that when the minimal repair probability $q$ increases, the minimum mean cost rate diminishes, and the optimal preventive replacement schedule extends. This is intuitive, as the lower probability of 
replacement leads to a lower mean cost and a lengthened replacement schedule.

- We see that some improvement can be made in the minimum mean cost rate if one allows for minimal repair at minor failure. That is to compare the maintenance policies proposed in this paper with pure maintenance policies (i.e., without minimal repair), minimal repair can lead to more advantage in maintenance cost.

- It can be seen that the present model is a generalization of the previous models and the policy with preventive replacement outperforms the one without preventive replacement.

\section{ACKNOWLEDGMENT}

This research was supported by the National Science Council of Taiwan, ROC, under Grant No. NSC102-2410-H147-001.

\section{REFERENCES}

[1] R.E. Barlow and L.C. Hunter, "Optimum preventive maintenance policies," Operations Research, vol. 8, pp. 90-100, 1960.

[2] H. Pham and H. Wang, "Imperfect maintenance," European Journal of Operational Research, vol. 94, pp. 425-438, 1996.

[3] M. Brown and F. Proschan, "Imperfect repair," Journal of Applied Probability, vol. 20, pp. 851-859, 1983.

[4] H.W. Block, W. Broges, ans T.H. Savits, "Age-dependent minimal repair,” Journal of Applied Probability, vol. 22, pp. 370-386, 1985.
[5] C.C. Chang, S.H. Sheu, and Y.L. Chen, "Optimal number of minimal repairs before replacement based on a cumulative repair-cost limit policy,” Computers \& Industrial Engineering, vol. 59, pp. 603-610, 2010.

[6] C.C. Chang, S.H. Sheu, and Y.L. Chen, "Optimal replacement model with age-dependent failure type based on a cumulative repair-cost limit," Applied Mathematical Modelling, vol. 37, pp. 308-317, 2013.

[7] Y.L. Chen, "A bivariate optimal imperfect preventive maintenance policy for a used system with two-type shocks," Computers \& Industrial Engineering, vol. 63, pp. 1227-1234, 2012.

[8] T. Sugiura, S. Mizutani, and T. Nakagawa, "Optimal random replacement policies," Tenth ISSAT International Conference on Reliability and Quality Design, pp. 99-103, 2004.

[9] W.Y. Yun and C.H. Choi, "Optimum replacement intervals with random time horizon," Journal of Quality in Maintenance Engineering, vol. 6, pp. 269-274, 2000.

[10] W. Stadje, "Renewal analysis of a replacement," Operations Research Letters, vol. 31, pp. 1-6, 2003.

[11] T. Nakagawa, Maintenance Theory of Reliability, Springer: Londen, 2005.

[12] M. Chen, S. Mizutani, and T. Nakagawa, "Random and age replacement policies," International Journal of Reliability, Quality and Safety Engineering, vol. 17(1), pp. 27-39, 2010.

[13] M. Chen, S. Nakagawa, and T. Nakagawa, "Replacement and Preventive maintenance models with random working times," IEICE Transactions on Fundamentals of Electronics, Communications and Computer Science, vol. 93-A, pp. 500-507, 2010.

[14] F. Beichelt, "A unifying treatment of replacement policies with minimal repair,” Naval Research Logistics, vol. 40, pp. 51-67, 1993.

[15] R.W. Drinkwater and N.A.J. Hastings, "A economic replacement model," Operational Research Quarterly, vol. 18, pp. 121-138, 1967.

[16] N.A.J. Hastings, "The repair limit replacement method," Operational Research Quarterly, vol. 20, pp. 337-349, 1969. 